\title{
Modeling of Prioritized Admission Control Supporting Adaptive Performance Management in Integrated Voice/Data CDMA Systems
}

\author{
Kuo-Chung $\mathrm{Chu}^{*}$ and Frank Yeong-Sung Lin ${ }^{\dagger}$ \\ Department of Information Management \\ National Taiwan University \\ Taipei, 106, Taiwan ROC $_{4}$ \\ e-mail: "d5725003@im.ntu.edu.tw, yslin@im.ntu.edu.tw
}

\begin{abstract}
Previous studies for CDMA admission control ignored traffic asymmetry analysis in both uplink and downlink. In this paper, we propose an admission control-based performance management framework for uplink and downlink connection in integrated voice/data CDMA systems. Based upon reserved channels (guard channels), the prioritized admission control problem is formulated as a performance optimization model. The objective is to minimize handoff forced termination probability. For solving the optimization problem, iteration-based Lagrangean relaxation approach is applied. This work provides both short-term and real-time admission control for updating system parameters and resource management, respectively.
\end{abstract}

Keywords- adaptive performance management; admission control; CDMA; Lagrangean relaxation; traffic asymmetry

\section{INTRODUCTION}

Demand for wireless communications and Internet applications is continuously growing. Due to the advantages in user capacity and soft handoff, code division multiple access (CDMA) has been proposed as the major multiple-access technique for the third generation ( $3 \mathrm{G})$ wireless systems. To support multiple classes of traffic/service, each traffic class is distinguished from others by its data rate and required quality of service (QoS). 3G systems provide a high-capacity mobile communications service.

Capacity analysis of previous researches has been conducted for the uplink connection [1][2][3][4], because the non-orthogonality leads to the limited capacity is in the uplink [5]. However, asymmetric internet traffic has increased and power allocation in a downlink is an important issue. In terms of power control, since the downlink capacity for a base station (BS) is limited by the maximum transmit power, which is a common resource to be shared by all mobile stations (MSs), a MS requiring a large fraction of this power could reduce the overall capacity. Theoretically, capacity is unbalanced on the downlink and uplinks. A number of reasons are identified [6] to show capacity is the downlink bottleneck, including traffic demands, handoff issue, interferences, antenna diversity.

Soft handoff is another characteristic in CDMA. During a soft handoff, the MS maintains simultaneous connections with more than one BS. The MS is allocated a downlink channel at each BS, and the information transmitted on each channel is the same. The MS performs diversity combining of the downlink paths, regardless of their origin.

Rejection of a soft handoff calls results in forced termination of an ongoing service. Thus, no matter what kind of service classes, soft handoff calls are more important than new call requests. There have been many studies regarding efficient call admission control (CAC) schemes that give priority to handoff calls over new calls, and can be classified into two groups. The first type reserves channels for handoff requests, and the second type queues handoff call requests waiting for available channels. Channel reservation scheme is useful since it gives priority to handoff requests, but it reduces handoff failure (forced termination) probability at the expense of a higher new call blocking probability. However, these studies ignored asymmetry traffic [1][7][8].

In consideration of performance management in $3 \mathrm{G}$ systems, adaptive admission control is dynamically monitoring system pattern in such a way to provide variable data control [8]. Therefore, each admitted service is guaranteed good performance while at the same time the system is operated at its maximum capacity. In this paper, we propose an admission control model for both uplink and downlink connection in integrated voice/data CDMA system, which supports traffic asymmetry with different QoS requirements on data rates and received signal-to-interference ratio (SIR). Based upon reserved channels (guard channels), the prioritized admission control problem is formulated as an optimization model. The objective is to minimize the handoff forced termination probability subject to new call blocking probability. The remainder of this paper is organized as follows. Section II describes the admission control problem and the concept of admission control-based adaptive performance management. In Section III, we illustrate relative issues for both uplink and downlink CDMA admission control, and propose prioritized admission control model in mathematical formulation. Section IV presents solution approach for optimal admission control model. Finally, the paper is concluded with Section V. 


\section{AdMISSION CONTROL PROBLEM OF CDMA NETWORKS}

\section{A. Previous Researches}

Generally, call requests can be categorized into both real new calls that are initiated in original BSs and handoff calls that are coming from adjacent BSs. Three CAC cases are presented to analyze voice only revenue contributions, 1) real new users are considered, they are admitted or rejected in its homing base station [9]; 2) only real new users are also considered, but they are admitted, rehomed to adjacent cells, or rejected $[10]$; 3) both real new calls and existing calls are taken into account, and forced handoff of existing calls can be conducted to optimally contribute overall revenue [11]. However, integrated voice/data service is a basic requirement in $3 \mathrm{G}$ systems. Even many studies focused on this issue, they provide no comprehensive model. Handoff call is not dealt with [2][4], without consideration of traffic asymmetry [3][4]. Besides, be more generic, giving priority to handoff calls, asymmetric traffic (both uplink and downlink) analysis, and heterogeneous service among BSs should be taken into account.

\section{B. Admission Control-based Adaptive Performance Management}

In consideration of performance management in $3 \mathrm{G}$ wireless networks, Lindemann et al. [8] proposed an adaptive performance management framework to improvement bandwidth utilization. The basic idea is to dynamically adjusting the packet scheduler and admission controller by means of a performance management information base ( $\mathrm{P}$ $\mathrm{MIB}$ ). In this research, we propose an admission control-based adaptive performance management framework in Figure 1, which is built in base station controller (BSC). There are three modules: performance optimization (admission control based), online performance monitoring, adaptive performance servicing.

1) Performance optimization module: This consists of an admission control optimization model which is described in Section III-D. Based on system settings (thresholds and parameters), arriving call requests are regulated to optimize performance measures (described in Section III-C). The detail of admission control architecture is illustrated in Figure 2; it admits call requests by channel management, in which guard channels $C_{j}^{\mathrm{g}}$ are reversed for handoff calls with higher priority, while ordinary channels $C_{j}^{o}$ are shared by all class types.

2) Online performance monitoring module: This module investigates the system pattern, including bit error rate (BER) of all class traffics, and channel utilization etc., in such a way to identifying performance bottlenecks. Tuning parameters in the servicing module to enhance the performance or resource relocation is needed otherwise. For example, capacity augmentation and expansion are prevalent approaches.

3) Adaptive performance servicing module: to optimal resource management, this module try to adjust system parameters, including $\operatorname{QoS}\left(E_{\mathrm{b}} / \mathrm{I}_{0}\right)$ thresholds for both links and a number of class traffics, fraction of reserved channels for handoff call requests. Alternatively, these updated parameters can be applied to real-time resource management for

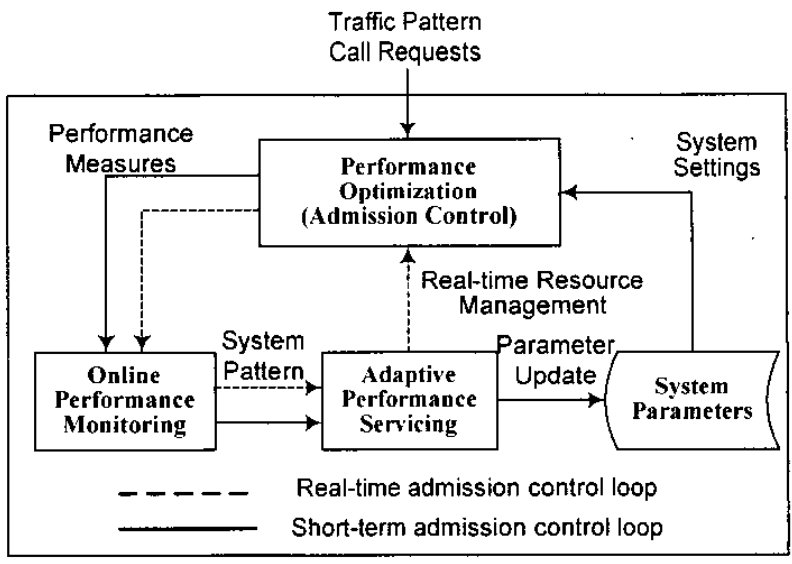

Figure 1. Adaptive performance management framework.

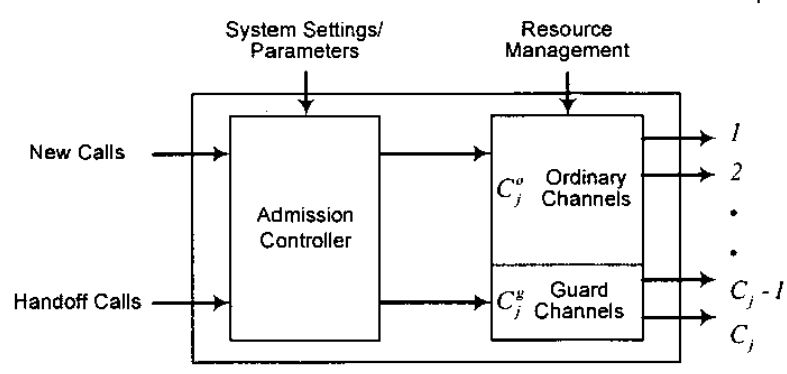

Figure 2. Admission control architecture.

dynamically adjusting data rates from released available resources.

Considering heterogeneous traffic demands in urban versus rural area, and irregular variations on demands, the parameters would be tuned weekly or monthly. For ongoing calls performance management, service level (data rate) can be promoted or degraded in terms of available resources released or granted, respectively. Changing service level is in seconds. In summary, this framework provides short-term and real-time admission control for updating system parameters and resource (data rate/channel) management, respectively.

\section{PRIORITIZED ADMISSION CONTROL SUPPORTING ASYMMETRY TRAFFIC}

\section{A. Traffic Model and Soft Handoff}

In this paper, we focus on voice and data traffics as well as new and handoff call requests. All service traffics are generated in Poisson arrival process with $\lambda_{j}^{V}$ and $\lambda_{j}^{D}$ for voice and data calls in $\mathrm{BS} j$, respectively. Total traffic load in BS $j$ is $\lambda_{j}=\lambda_{j}^{r}+\lambda_{j}^{D}$. By the way, both $\lambda_{j}^{r}$ and $\lambda_{j}^{D}$ consists of new and handoff calls $\lambda_{j}^{V}=\lambda_{j}^{V, N}+\lambda_{j}^{V, H}$ and $\lambda_{j}^{D}=\lambda_{j}^{D, N}+\lambda_{j}^{D . H}$. Total new and handoff call in BS $j$ is $\lambda_{j}^{N}=\lambda_{j}^{V, N}+\lambda_{j}^{D, N}$ and $\lambda_{j}^{H}=\lambda_{j}^{r^{\prime}, H}+\lambda_{j}^{D, H}$, respectively.

Alternatively, in consideration of handoff call loading, it 
is in proportion to adjacent $\mathrm{BS} \lambda_{j}$ [7]. Be more realistic, for example, considering user soft handoff between two nearest BSs in cell 0 and cell 1 . User $t$ in cell 0 enters soft handoff when the received strength of the pilot from cell $1\left(p_{1 i}\right)$ is greater than that from cell $0\left(p_{0 t}\right)$ minus $\Delta d B$. The soft handoff status remains until $p_{1 i}$ exceeds $p_{0 i}$ plus $\Delta d B$. Assume that in the soft handoff phase the two base stations transmit to the user with the same fraction $\phi_{j}$ of the BS $j$ power [13]. We combine [7][13] works to determine $\lambda_{j}^{H}$.

\section{B. SIR Models}

In CDMA environments, since all uses communicate at the same time and same frequency, each user's transmission power is regarded as a part of other users' interference. Thus, CDMA is a kind of power-constrained or interference-limited system. With perfect power control and the interference-dominated system, we ignore background noise $\left(N_{0}\right)$. Let $W_{U}$ and $W_{D}$ be the system bandwidth for uplink and downlink, respectively. Let $d_{s}^{U}$ and $d_{s}^{D}$ be the data rate of class- $s$ for uplink and downlink, respectively. Decision variable $z_{j t}^{s . y}$ is 1 if class-s's type y call of MS $t$ is admitted by BS $j$ or 0 otherwise.

1) Uplink SIR model: We assume that uplink power is perfectly controlled, it assure the received power at the BS is the same for all MSs. The number of class-s's new and handoff calls in BS $j$ are $n_{j}^{s, N}=\sum_{i \in T} z_{j t}^{s, N}$ and $n_{j}^{s . H}=\sum_{t \in T} z_{j t}^{s . H}$, respectively. Denote $P_{s}$ the received uplink power in BS by service class- $s$. Intracellular interference in uplink with respect to class-s is given by (1), where $\alpha_{s}^{U}$ is uplink class- $s$ activity factor.

$$
I_{\text {intra }}^{U}=P_{s}\left[\sum_{s \in S} \alpha_{s}^{J}\left(n_{j}^{s, H}+n_{j}^{s, H}\right)-1\right]
$$

For intercellular interference, total received power from other cells is given by (2), and denotes $D_{j t}$ distance from MS $t$ to $\mathrm{BS} j$. This idea stems from Eq. (15) in [12].

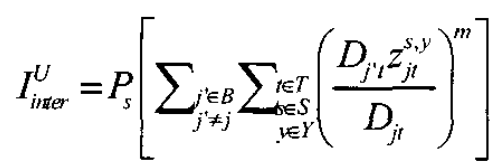

The received SIR of service class- $s$ at BS $j$ is given by

$$
S I R_{j, s}^{U}=\frac{W_{U}}{d_{s}^{U}} \frac{P_{S}}{\left(1-\theta^{U}\right) I_{\text {intra }}^{U}+I_{\text {inter }}^{U}}
$$

where $\theta^{v}$ is the uplink orthogonality factor.

2) Downlink SIR model: In downlink case, there is constraint on BS's total transmit power instead of individual MS. BS power is divided among the MSs communicating with

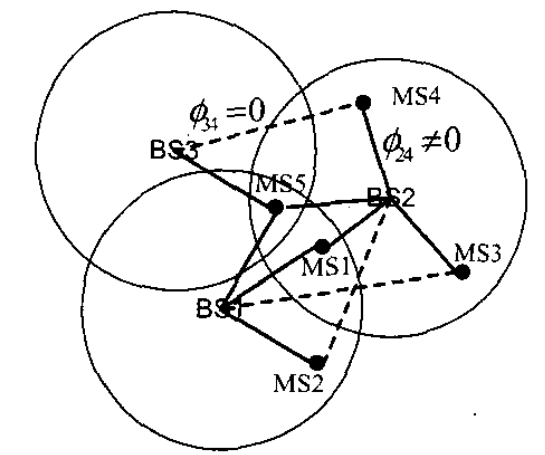

Figure 3. Downlink interferences representation by $\phi_{j r}$.

the BS and the overhead signals. Increasing the BS power increase the power per connection. Let $P_{j}^{T}$ and $L_{j t}$ be the total transmitted power from BS $j$ and path loss from $\operatorname{BS} j$ to $\mathrm{MS} t$, respectively. For a specific MS $t$, its received power $P_{t}$ from MS $j$ is given by (4), where $\phi_{j t}\left(\sum_{t \in T} \phi_{j t} z_{j t}=1, \forall j \in B\right)$ is the fraction of power transmitted to $\mathrm{MS} t$ from $\mathrm{MS} j, L_{j t}=D_{j t}^{-i} \cdot \chi_{j t}$, $l$ is path loss exponent [12].

$$
P_{t}=\sum_{j \in B} z_{j t} \phi_{j t} P_{j}^{T} L_{j t}
$$

Furthermore, applying $\phi_{j t}$ and $z_{j t}$, both downlink intracellular interference $I_{\text {intra }}^{D}$ and intercellular interference $I_{\text {inter }}^{D}$ can be by given by (5) and (6), respectively.

$$
\begin{aligned}
I_{i n t r a}^{D} & =\sum_{j \in B}\left(1-\phi_{j t}\right) z_{j t} P_{j}^{T} L_{j t} \\
I_{i n t e r}^{D} & =\sum_{j \in B}\left(1-z_{j t}\right) \phi_{j t} P_{j}^{T} L_{j t}
\end{aligned}
$$

By integrating (4)-(6), we get the received SIR at MS $t$ in (7), where $\theta_{t}^{D}$ is the downlink orthogonality factor for MS $t$.

$$
S_{t} R_{t}^{D}=\frac{W_{D}}{d_{s}^{D}} \frac{P_{t}}{\left(1-\theta_{t}^{D}\right) I_{\text {intra }}^{D}+I_{\text {inter }}^{D}}
$$

Reducing the uplink data rate $d_{s}^{D}$ leads to higher processing gain which decreases power requirements. This results in reduced BS power and interferences. As a result, forward link capacity will increase.

In order to represent $I_{i n r u}^{D}$ and $I_{\text {inter }}^{D}$ by both $\phi_{i t}$ and $z_{j t}$, we show that by example in Figure 3. There are three BSs with five distributed MSs. The service condition of MSs is expressed by (8), in which the array element $z_{j t}$ is 1 if MS $t$ serviced by BS $j$ or 0 otherwise. From which, we get array of in (9). Some $\phi_{j t}$ are illustrated by solid-line with $\phi_{j t} \neq 0$, dash-line with $\phi_{j t}=0$ with respect to $z_{j t}$. 


$$
\begin{gathered}
z_{j t}=\left[\begin{array}{ccccc}
1 & 1 & 0 & 0 & 1 \\
1 & 0 & 1 & 1 & 1 \\
0 & 0 & 0 & 0 & 1
\end{array}\right] \\
\phi_{j t}=\left[\begin{array}{ccccc}
\phi_{11} & \phi_{12} & 0 & 0 & \phi_{15} \\
\phi_{21} & 0 & \phi_{23} & \phi_{24} & \phi_{25} \\
0 & 0 & 0 & 0 & \phi_{35}
\end{array}\right]
\end{gathered}
$$

\section{Performance Measure}

Admission control is based on SIR measurement. However, providing guaranteed QoS for ongoing calls is more important than admitting new call requests. Based on SIR threshold, if we give priority to new calls, the forced termination of ongoing calls degrades service level. Due to the soft handoff advantage in CDMA system, we would like to focus on minimization of handoff call forced termination (blocking) probability subject to given new call blocking probability. Channel reservation scheme is useful since it gives priority to handoff requests. The reserved $C_{j}^{g}\left(=\left\lceil C_{j} \cdot f_{j}\right\rceil\right.$, a ceiling function) channels among $C_{j}$ channels available in BS $j$ are referred to as the guard channels, where $f_{j}$ is a reserved ratio of $C_{j}$ and is be determined. The remaining $C_{j}^{o}\left(=C_{j}-C_{j}^{g}\right)$ channels, called the ordinary channels, are shared by both calls [1][7][8]. When a new call attempt is generated in $\mathrm{BS} j$, it is blocked if the number of free channels is less than or equal to $C_{j}^{g}$. Then the blocking probabilities of new and handoff calls in the $\mathrm{BS} j$ are given by (10) and (11) [1], respectively.

$$
\begin{aligned}
& B N_{j}\left(\lambda_{j}^{N}, \lambda_{j}^{H}, C_{j}^{o}, C_{j}^{g}\right)= \\
& \frac{\left(\lambda_{j}\right)^{C_{i}^{o}} / C_{j}^{o} !+\left(\lambda_{j}\right)^{C_{j}^{o}} \sum_{c=1}^{C_{j}^{o}}\left(\lambda_{j}^{H}\right)^{C} /\left(C_{j}^{o}+c\right)}{\sum_{C=0}^{C_{j}^{o}}\left(\lambda_{j}\right)^{C} / c !+\left(\lambda_{j}\right)^{C_{j}^{o}} \sum_{c=1}^{C_{j}^{s}}\left(\lambda_{j}^{H}\right)^{C} /\left(C_{j}^{o}+c\right)} \\
& B H_{j}\left(\lambda_{j}^{H}, \lambda_{j}^{H}, C_{j}^{o}, C_{j}^{\mathrm{g}}\right)= \\
& \frac{\left(\lambda_{j}\right)^{C_{j}^{o}}\left(\lambda_{j}^{H}\right)^{c_{j}^{o}} /\left(C_{j}^{v}+C_{j}^{g}\right) !}{\sum_{C=0}^{C_{i}^{o}}\left(\lambda_{j}\right)^{C} / c !+\left(\lambda_{j}\right)^{C_{j}^{o}} \sum_{C=1}^{C_{j}^{g}}\left(\lambda_{j}^{H}\right)^{C} /\left(C_{j}^{o}+c\right)}
\end{aligned}
$$

To minimize the handoff call forced termination probability, the weighted probability is given by

$$
Z_{I P}=\sum_{j \in B} w_{j} B H_{j}\left(\lambda_{j}^{N}, \lambda_{j}^{H}, C_{j}^{o}, C_{j}^{g}\right)
$$

where $w_{j}=\lambda_{j}^{H} / \sum_{j \in B} \lambda_{j}^{H}$

\section{Prioritized Admission Control Modeling}

Based on discussions, we consider overall system consisting of $B$ BSs and $T$ MSs with service class set $S$, voice and data calls. Each of calls fits into call type set $Y$, including real new call and handoff call. Decision variable $z_{j t}^{s, y}$ is 1 if class- $s$ 's type y call of MS $t$ is admitted by BS $j$ or 0 otherwise. The overall performance optimization problem is (IP) in terms of admission control. The objective is to minimize handoff forced termination probability subject to new call blocking probability.

$$
\min \sum_{j \in B} w_{j} B H_{j}\left(\lambda_{j}^{N}, \lambda_{j}^{H}, C_{j}^{o}, C_{j}^{g}\right)
$$

s.t.

$$
\begin{gathered}
\left(\frac{E_{b}}{I_{0}}\right)^{U} \leq S I R_{j, s}^{U} \forall j \in B, s \in S \\
\left(\frac{E_{b}}{I_{0}}\right)^{D} \leq S I R_{t}^{D} \forall j \in B, t \in T \\
\sum_{s \in S} z_{j t}^{s, H} \delta_{j t}^{s, H} \leq C_{j} \forall j \in B, t \in T \\
\sum_{s \in S} z_{j t}^{s, N} \delta_{j t}^{s, H} \leq C_{j}^{o} \forall j \in B, t \in T \\
\sum_{y \in Y} z_{j t}^{s, y} \leq 1 \quad \forall j \in B, t \in T, s \in S \\
z_{j t}^{s, y} D_{j t} \leq R_{j} \forall j \in B, t \in T, y \in Y, s \in S \\
z_{j t}^{s, y} \leq \delta_{j t}^{s, y} \forall j \in B, t \in T, y \in Y, s \in S \\
P_{j}^{T} L_{j t} \geq\left(\varepsilon_{j}+P_{j^{\prime}}^{T} L_{j \prime}\right) \delta_{j t}^{s . H} \forall j \in B, t \in T, s \in S \\
B N_{j}\left(\lambda_{j}^{N}, \lambda_{j}^{H}, C_{j}^{o}, C_{j}^{g}\right) \leq \beta_{j} \forall j \in B, t \in T \\
z_{j t}^{s, H} \geq z_{j t}^{s, N} \forall j \in B, t \in T, t \in T, s \in S, t \neq t \\
0 \leq P_{j}^{T} \leq P_{M A X} \forall j \in B \\
z_{j t}^{s, y}=0 \text { or } 1 \forall j \in B, t \in T, y \in Y, s \in S
\end{gathered}
$$

SIR constraint for uplink and downlink is (13) and (14), respectively. Constraints (15) and (16) are to ensure available channel for handoff and new calls, respectively. Denote $\delta_{j t}^{s, y}$ indication function is 1 if MS $t$ initiates class-s's type $y$ call in MS $j$, or 0 otherwise. Constraint (17) is to guarantee only one of call types is admitted for MS. MS can be serviced in the coverage of BS by (18), where $R_{j}$ and $D_{j t}$ is the power transmission radius and the distance to MS. Constraint (19) implies a call can be admitted only if the call is initiated. Constraint (20) decides if handoff call is initiated by a threshold $\Delta d B=\varepsilon_{j}$ described in Section III-B. The threshold $\beta_{j}$ of new call blocking probability in $\mathrm{BS} j$ is also given in (21). Prioritized admission control is shown in (22) by giving higher priority to handoff calls. Power transmission constraint is given in (23). Finally, constraint (24) ensures integral property.

\section{MODEL SOLUTION}

\section{A. Lagrangean Relaxation Approach}

Applying Lagrangean relaxation method is described in [15] [16]. Problem (IP) is transferred to be a dual problem (D) 
by relaxing $m$ complicating constraints, then multiply the relaxed constraints with corresponding Lagrangean multipliers vector $V=\left(v_{l}, v_{2}, \ldots \ldots v_{m}\right)$, and add them to the primal objective function. According to the weak Lagrangean duality theorem [15], for any $V \geq 0$, the objective value of $Z_{D}(V)$ is a lower bound of $Z_{I P}$. Thus, the following dual problem (D) is constructed to calculate the tightest lower bound by adjusting multipliers.

$$
Z_{D}=\max Z_{D}(V)
$$

subject to: $V \geq 0$. Then, subgradient method [17] is applied to solving the dual problem. Let the vector $S$ is a subgradient of $Z_{D}(V)$ at $V \geq 0$. In iteration $k$ of subgradient optimization procedure, the multiplier vector $\pi$ is updated by $\pi^{k+1}=\pi^{k}+t^{k} S^{k}$, in which $t^{k}$ is a step size determined by $t^{k}=\lambda\left(Z_{I P}^{*}-Z_{D}\left(\pi^{k}\right)\right) /\left\|S^{k}\right\|^{2}$, where $Z_{I P}^{*}$ is an upper bound on the primal objective function value after iteration $k$, and $\lambda$ is a constant where $0 \leq \lambda \leq 2$.

\section{B. Real-time Resource Management}

1) Real-time rationale of Lagrangean relaxation: Based upon Lagrangean relaxation approach, a predefined time budget, e.g. 10 seconds of time period for real-time requirement, is given to solving Lagrangean dual problem and getting primal feasible solutions iteratively. Number of iterations is depended on the time budget. On the other hand, initial values of Lagrangean multipliers and upper bound affect solution quality on algorithm convergence. If we appropriately assign initial values, algorithm will be speeded up to converge in stead of more iterations are required.

\section{2) Algorithm:}

- Step 1: Generate number users arrived in the consecutive time periods.

Step 2: Apply Lagrangean relaxation procedure to solve problem (IP) for each time period

Step 2.1: Set initial values of multipliers, for each Lagrangean procedure.

Step 2.2: Solve Lagrangean dual problem (D) for lower bound.

Step 2.3: Get primal feasible solutions for upper bound in considering relaxed constraints.

Step 2.3: Update bounds and multiplier vector $\pi$.

Step 2.4: Compute $Z_{i p \text {. }}$

Step 2.5: Iterate steps 2.1 to 2.4 until solution gap converge or time budget expire otherwise.

Step 3: Analyze performance measure.

Step 4: Iterate steps 2 and 3, until end of predefined number of time periods are experimented.

\section{CONCLUSIONS}

This paper proposes a prioritized admission control model to supporting asymmetry traffic in integrated voice/data CDMA systems. We jointly consider uplink/downlink, new/handoff calls. Based on the admission control, we also construct an adaptive resource management framework to achieve overall performance optimization. For solving the optimization problem, iteration-based Lagrangean relaxation approach is applied. This work provides short-term and realtime admission control for updating system parameters and resource (data rate/channel) management, respectively. Other two modules (online performance monitoring, adaptive performance servicing) and numerical analysis will be specified in the future work.

\section{REFERENCES}

[1] Kun-Nyeong Chang and Dongwoo Kim, "Optimal Prioritized Channel Allocation in Cellular Mobile Systems," Computers \& Operations Research, vol. 28, pp. 345-356, 2001.

[2] Hwa-Young Um and Soon-Yong Lim, "Call Admission Control Schemes for DS-CDMA Cellular System Supporting an Integrated Voice/Data Traffic," in Proc. IEEE ISCC. pp. 365-369, 1998.

[3] Wha Sook Jeon, and Dong Geun Jeong, "Call Admission Control for CDMA Mobile Communications Systems Supporting Multimedia Services," IEEE Trans. Wireless Communications, vol. 1, no. 4, Oct. pp. 649-659, 2002.

[4] Wen-Bin Yang and Evaggelos Geraniotis, "Admission Policies for Integrated Voice and Data Traffic in CDMA Packet Radio Networks," IEEE J. Select. Area Commun., vol. 12, no. 4, pp. 654664, May 1994.

[5] Audrey M. Vitervi, Andrew J. Vitervi, "Erlang Capacity of a power controlled CDMA system," IEEE J. Select. Area Commun., vol. 11, no. 6, pp. 892-900, Aug., 1993.

[6] Jaber A, Khoja and Jonathan W. Bredow, "Strategy to Increase Forward Link Capacity of 3G CDMA Wireless Networks by QOS (Data-Rate) Modification," IEEE Radio and Wireless Conference (RAWCON), pp. 1-4, 2002.

[7] Novella Bartolini and Imrich Chlamtac, "Call Admission Control: Solution of a General Decision Model with State Related Hand-off Rate," in Proc. IEEE WCNC, pp. 237-241, 2000.

[8] Christoph Lindemann, Marco Lohmann, Axel Thummler, "Adaptive performance management for universal mobile telecommunications system networks," Computer Networks, vol. 38, pp. 477-496, 2002.

[9] Kuo-Chung Chu, Frank Yeong-Sung Lin, and Shin-Fu Wang, "An admission control algorithm for CDMA networks," accepted for publication, in Proc. 1lth IEEE ICON, pp. 647-652, 2003.

[10] Kuo-Chung Chu, Frank Yeong-Sung Lin, and Shin-Fu Wang, "Performance Analysis of Admission Control Algorithms for CDMA Networks," in Proc. 5th IFIP MWCN, pp. 285-288, 2003.

[11] Kuo-Chung Chu, Frank Yeong-Sung Lin, and Shin-Fu Wang, An Admission Control Algorithm for Revenue Optimization CDMA Networks," in Proc. 11th IEEE ISPACS, pp. 763-768, 2003.

[12] Wai-Man Tam; Lau, F.C.M., "Analysis of power control and its imperfections in CDMA cellular systems," IEEE Trans. Veh. Technol., vol. 48, no. 5, pp. 1706 - 1717, Sept. 1999.

[13] Lei Zhuge and Victor $\mathrm{O} . \mathrm{K}$. Li, "Forward Link Capacity in Multiservices DS-CDMA Systems," in Proc. IEEE GLOBECOM, pp. 609-613, 2001.

[14] Wan Choi and Jin Young Kim, "Forward-Link Capacity of a DSCDMA System with Mixed Multirate Sources," IEEE Trans. Veh. Technol., vol. 50, no. 3, pp. 737-749, May 2001.

[15] A. M. Geoffrion, "Lagrangean relaxation and its use in integer programming", Math. Programming Study, vol. 2, pp. 82-1 14, 1974.

[16] M. L. Fisher, "The Lagrangian relaxation method for solving integer programming problems", Management Science, vol. 27, pp. 1-18, 1981

[17] M. Held, P. Wolfe, and H. D. Crowder, "Validation of subgradient optimization", Math. Programming, vol. 6, pp. 62-88, 1974. 